\title{
Study of Swift Heavy Ion Modified Conducting Polymer Composites for Application as Gas Sensor
}

\author{
Alok Srivastava ${ }^{1,2,{ }^{*}}$, Virendra Singh ${ }^{1}$, Chetna Dhand ${ }^{1}$, Manindar Kaur ${ }^{1}$, Tejvir Singh ${ }^{1}$, \\ Karin Witte $^{2}$ and Ulrich W. Scherer ${ }^{2}$ \\ ${ }^{1}$ Department of Chemistry, Panjab University, Chandigarh-160014, India \\ ${ }^{2}$ Aachen University of Applied Sciences, Jülich Campus, Ginsterweg 1, 52428 Jülich, Germany \\ * Author to whom correspondence should be addressed. Email: alok@pu.ac.in
}

Received: 29 August 2005 / Accepted: 04 January 2004 / Published: 7 April 2006

\begin{abstract}
A polyaniline-based conducting composite was prepared by oxidative polymerisation of aniline in a polyvinylchloride (PVC) matrix. The coherent free standing thin films of the composite were prepared by a solution casting method. The polyvinyl chloride-polyaniline composites exposed to $120 \mathrm{MeV}$ ions of silicon with total ion fluence ranging from $10^{11}$ to $10^{13}$ ions $/ \mathrm{cm}^{2}$, were observed to be more sensitive towards ammonia gas than the unirradiated composite. The response time of the irradiated composites was observed to be comparably shorter. We report for the first time the application of swift heavy ion modified insulating polymer conducting polymer (IPCP) composites for sensing of ammonia gas.
\end{abstract}

Keywords: Conducing polymer, ammonia gas sensors, swift heavy ions, polymer composites. 


\section{Introduction}

Conducting polymers have attracted considerable interest due to their various applications in organic light-emitting diodes (OLEDs) [1], lithography [2], electrode material [3], electromagnetic shielding [4] and sensing [5-6]. Sensors made from conducting polymers as varied as polypyrrole, polyhexylthiophene, polymethylthiophene, polyethylene and polyaniline have been investigated [7-12] to study their potential application in detecting gases, like nitrogen dioxide, carbon monoxide, ammonia, and hydrogen besides moisture and a number of organic volatile compounds. The conducting polymer polyaniline is known for its ease in synthesis and environmental stability. Its redox behaviour (doped/dedoped) makes it a potential candidate for chemical sensing. However, its poor solubility and processability limits its applications. To overcome these problems various attempts have been made to blend polyaniline with insulating polymers that have good mechanical strength [6]. The composites of polyaniline have been prepared successfully by chemical [13] and electrochemical [14] polymerisations in presence of the insulating matrix.

The modification of polymeric material by irradiation with swift heavy ions [15-16] has gained considerable interest in the last two decades due to easy access to heavy ion accelerators. Studies pertaining to the study of the effect of gamma and electron irradiation [17] and swift heavy ions [18] on the electrical properties of polyvinyl chloride-polyaniline (PVC-PANI) blends have been reported in literature. On irradiation with swift heavy ions, a dramatic change in the electrical properties of polyaniline composites was observed [18]. Swift heavy ion irradiated polyvinylchloridepolyethylenterephthalate (PVC-PET) composites have been tested for hydrogen gas sensitivity [19]. In the present study, we report the method for the preparation of polyvinylchloride-polyaniline composite free standing films and the effect of swift heavy ions on the sensing behaviour of the composite films.

\section{Experimental}

\section{Materials}

Aniline, ammonium per-oxodisulphate (APS), tetrahydrofuran (THF), and ammonia solution were of analytical grade from E. Merck. Aniline was distilled under reduced pressure in presence of zinc dust prior to use. The PVC powder, with an average degree of polymerization of 1800 to 2500, was procured from Aldrich. All other chemicals were used as received.

\section{Polymerization of aniline in presence of PVC powder}

Freshly distilled aniline was oxidized with APS in the presence of PVC powder in hydrochloric acid medium. Aniline monomer dissolved in $\mathrm{HCl}(1 \mathrm{M})$ was stirred and brought to the desired temperature (-5 to $5{ }^{0} \mathrm{C}$ ). PVC powder was dispersed in the aniline hydrochloride solution and kept stirring for $1 \mathrm{hr}$. APS solution in hydrochloric acid (1 M), maintained at the same temperature, was added drop wise to start polymerization. During the polymerization the solution was kept in a salt ice bath to maintain the temperature in the desired range. After a few minutes PVC particles turned from white to green and then dark green. 
The reaction completed within a period of 4 hrs and the product was collected on Buchner funnel. The collected product was washed with deionized water and alcohol until the filtrate turned colorless. The product was dried in a vacuum desiccator. The weight ratio of PVC to aniline was kept 90.

\section{Preparation of PANI-PVC films}

The composite powder was washed with ammonia solution $5 \%(\mathrm{v} / \mathrm{v})$ and doped by stirring with camphor sulphonic acid (CSA) solution for 12 hrs. The CSA doped conductive composite powder was soluble in freshly dried THF (14). Conductive composite (1 g) was dissolved in dry THF (40 mL) and stirred in a graduated beaker until $20 \mathrm{~mL}$ of the volume of solution was reduced. The viscous solution was poured in a petriplate (diameter $100 \mathrm{~mm}$ ) and kept under ambient temperature. The solvent evaporated after $24 \mathrm{hrs}$ and a thin film $(40 \mu \mathrm{m})$ was peeled out from petriplate.

\section{Irradiation}

The films were exposed to $120 \mathrm{MeV}$ silicon ions in the material science facility of the $15 \mathrm{UD}$ pelletron at the Nuclear Science Centre, New Delhi. The irradiation of the films was carried out at room temperature and under vacuum $\left(10^{-6}\right.$ Torr $)$ for time periods ranging from 120 to 1200 seconds. The thickness of the film $(40 \mu \mathrm{m})$ is less than the ion projected range so as to avoid any complexity due to ion implantation. The ion projected range in PVC-PANI (90:10) for $120 \mathrm{MeV}$ Si ions was calculated as $50.8 \mu \mathrm{m}$ using the SRIM 2003 [20]. The total numbers of ions falling on the target were estimated to be in the range of $10^{11}$ to $10^{13}$ ions $/ \mathrm{cm}^{2}$ depending on the ion current and the time of bombardment. The ion beam was defocused using magnetic scanning system, so that an area of $1 \times 1 \mathrm{~cm}^{2}$ was uniformly irradiated. The beam current was kept low and was monitored intermittently with a Faraday cup.

\section{Electrode preparation and gas sensing}

The surface-free electrode is essential for measuring the chemical sensitivity of conducting polymer composites for their application within sensors. Both pristine and irradiated films were coated with $\mathrm{Al}$ using vacuum deposition method (Fig. 1a). To these films two leads of copper wire were attached with silver paste. The wires were connected to a digital electrometer (Keithley 2000). These electrodes were fixed in a home-made closed pyrex glass gas chamber (Fig. 1b) and exposed to the vapours of ammonia with different concentrations (v/v). The sensitivity factor was monitored in terms of the normalized resistance $(\mathrm{R} / \mathrm{Ro})$, where $\mathrm{R}$ is the measured resistance after exposure to ammonia vapours and Ro is the initial resistance. 


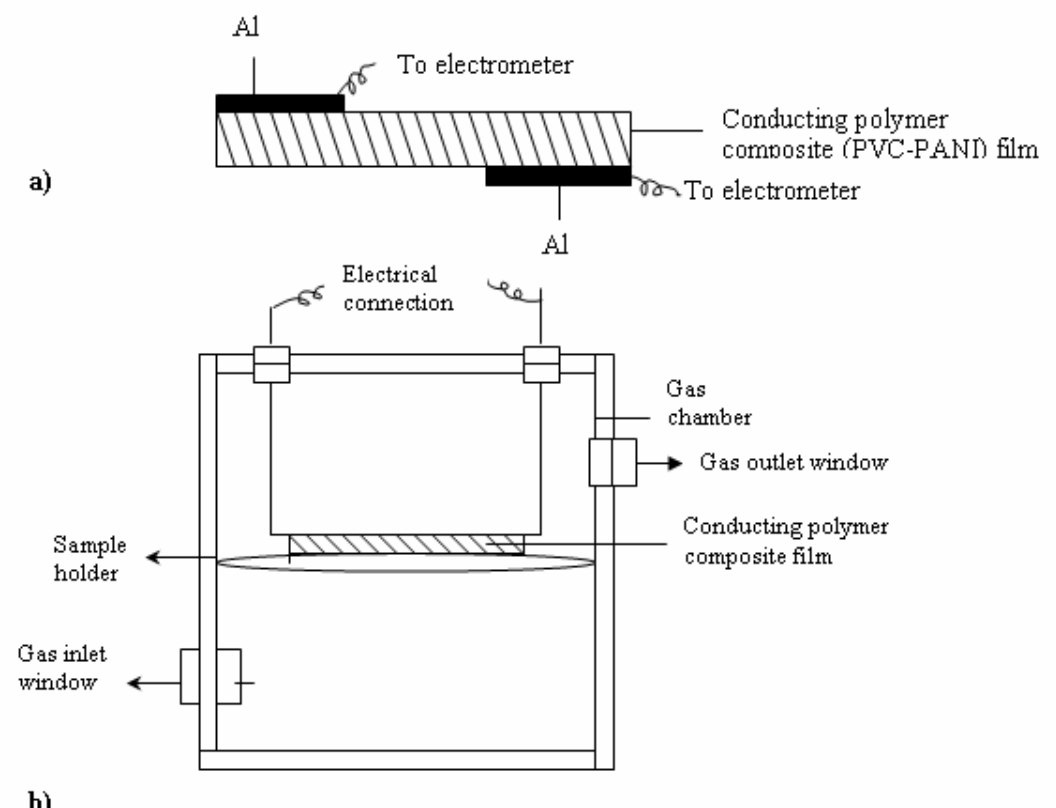

Figure 1. Schematic diagram of (a) electrode and (b) experimental setup used for sensitivity measurements.

\section{Results and discussion}

Spectroscopic investigations

Figure 2 shows the Fourier-transformed infrared (FT-IR) spectra of pure PVC and the PVCPolyaniline composite. A comparison of Figures 2a and 2b shows the appearance of new peaks at 3440, 1596 and $1504 \mathrm{~cm}^{-1}$ due to the stretching vibration of $\mathrm{N}-\mathrm{H}$, quinoid ring and benzenoid rings, respectively [21]. This indicates the formation of a PVC-PANI composite. The intensity of the stretching bands of pure PVC were found to decrease in the composite. The decrease in the intensity of PVC bands was due to the decrease in the PVC contents as a result of the interaction of PVC contents with the nucleophilic imine part of polyaniline.

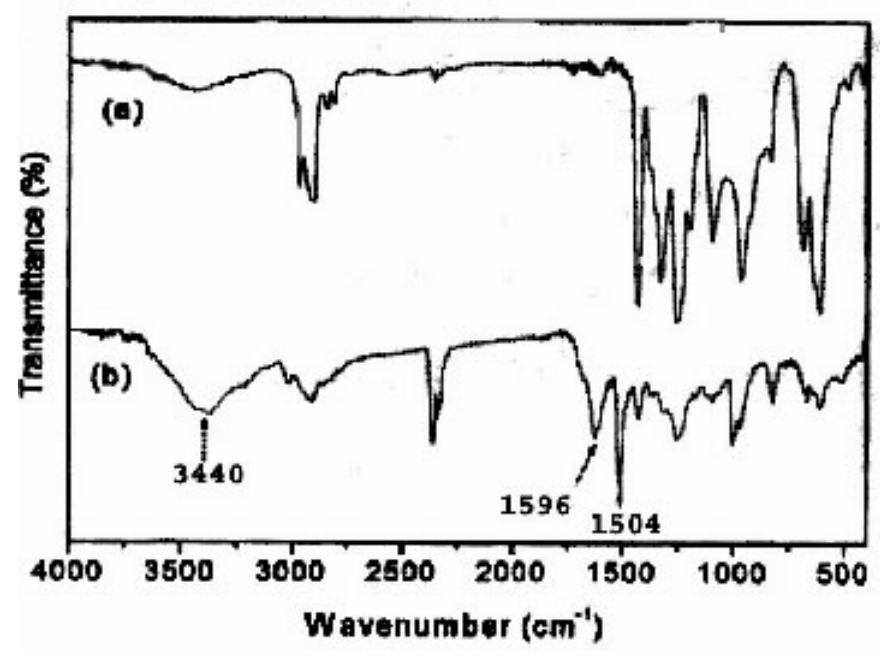

Figure 2. FT-IR spectra of (a) pure PVC and (b) PVC-Polyaniline composite. 


\section{Sensing activity}

Polyaniline is a unique polymer and shows a reversible acid/base doping process. In the acid doped or emeraldine salt (ES) form, polyaniline is conductive, while in the emeraldine base (EB) or dedoped form, this polymer is insulating. The emeraldine salt on treatment with base (ammonia vapors) can be converted to emeraldine base, which on reaction with acid ( $\mathrm{HCl}$ vapors) gets reconverted to emerladine salt. Thus, two forms are interconvertable as shown:

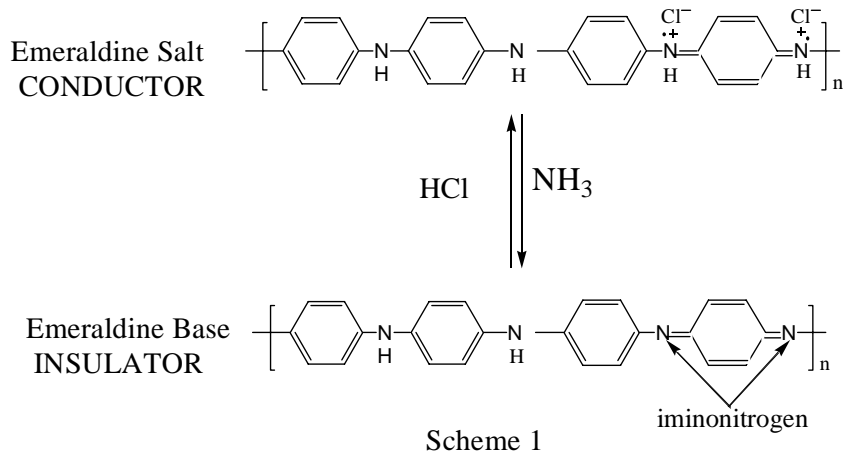

The detection sensitivity of the sensors was determined by studying the surface resistance as a function of the concentration of ammonia gas under similar ambient condition. The surface resistance of the films both pristine and the irradiated was plotted as a function of the concentration of ammonia gas in percent volume in Figure 3.

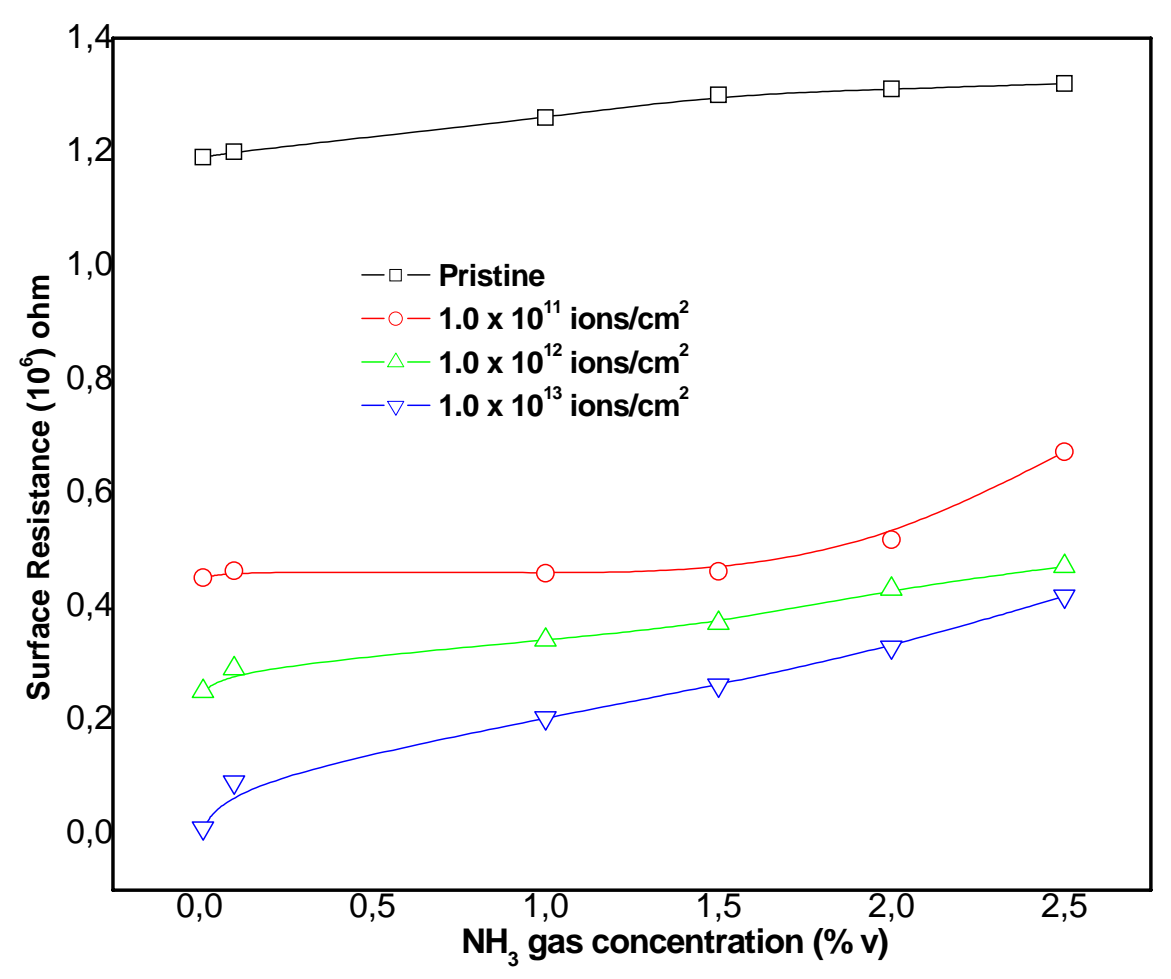

Figure 3. Plot of surface resistance as a function of ammonia gas concentration at different ion fluence.

It is clearly observed from Figure 3 that the surface resistance of the films decreases with increasing ion fluence at any given concentration of ammonia and is reduced by nearly two orders of magnitude $\left(10^{6} \mathrm{ohm}\right.$ to $\left.10^{4} \mathrm{ohm}\right)$ due to ion irradiation. It is further observed that the resistance of the irradiated 
samples exposed to different concentration of ammonia gas increased slightly more steeply than that of the pristine sample. The electrical response of the composite films exposed to ammonia is shown in Figure 4 in terms of the normalized resistance as a function of time for the pristine film and the films irradiated with total ion fluence of $1.0 \times 10^{12}$ and $1.0 \times 10^{13}$ ions $/ \mathrm{cm}^{2}$, respectively. The study on the temporal behaviour of normalized resistance indicates that the irradiated composite films have a slightly quicker response than that of pristine. It could be perhaps due to the irradiation-induced porosity in the composite films, which results in faster adsorption of ammonia vapours. As ammonia vapours interact with the electrophilic site of the polymer, it captures the protons followed by the chloride ions from iminonitrogen to form uncharged polymer (EB form) and ammonium chloride. This results in an increase of the resistance of the polymer composite films on exposure to ammonia vapours. Therefore, it can be stated that the increased sensitivity and better response time in the case of irradiated composites could perhaps be due to the structural modification taking place in the polymer material as a result of heavy ion exposure, which leads to more efficient diffusion of ammonia gas in the composite matrix.

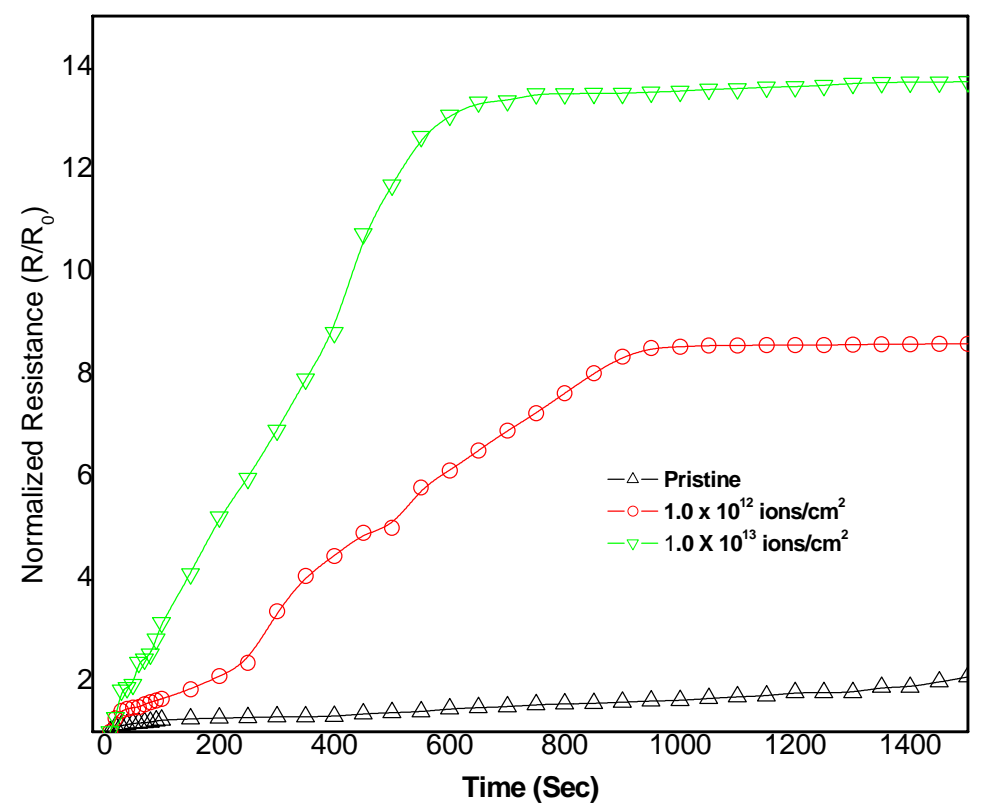

Figure 4. Electrical response of composite films to $1000 \mathrm{ppm}$ of ammonia gas.

\section{Conclusions}

The electrical conductivity of polymer composites show a significant change upon irradiation with swift heavy ion beam. The resistance is found to decrease with increase in ion fluence. The ion beam modified polymer composites are found to be more sensitive to ammonia gas. The sensitivity increased by nearly 100 times when the ion fluence was raised from $1.0 \times 10^{11}$ ions $/ \mathrm{cm}^{2}$ to $1.0 \times 10^{13}$ ions $/ \mathrm{cm}^{2}$. The response time was observed to be considerably shorter for the irradiated composites under the present experimental conditions. The present work is the first attempt to study the role of swift heavy ions in the gas sensing application of insulating polymer conducting polymer (IPCP) composites. 


\section{Acknowledgments}

The authors are grateful to the pelletron operating staff at NSC, New Delhi. V. Singh is thankful to UGC for providing a research fellowship. One of us (A.S) is thankful to German Academic Exchange Service (DAAD) and Alexander von Humboldt Foundation, Germany, for sponsoring his stay in Aachen University of Applied Sciences, Gemany.

\section{References}

1. Schultze, J.W.; Karabulut, H. Application potential of conducting polymers. Electrochimica Acta, 2005, 50, 1739.

2. Asmus, T.; Wolf, G. K. Modification and structuring of conducting polymer films on insulating substrates by ion beam treatment. Nucl. Instr. and Meth. in Phy. Res. B., 2000, 166, 732.

3. Mastragostino, M.; Arbizzani, C.; Soavi, F. Conducting polymers as electrode materials in super capacitors. Solid State Ionics, 2002, 148, 493.

4. Dhawan, S.K.; Singh, N.; Rodrigues, D. Electromagnetic shielding behaviour of conducting polyaniline composites. Science and Technology of Advanced Materials, 2003, 4, 105.

5. Koul, S.; Chandra, R.; Dhawan, S.K. Conducting polyaniline composite: a reusable sensor material for aqueous ammonia. Sensors and Actuators B: Chemical, 2001, 75, 151.

6. Jain, S.; Chakane, S.; Samui, A.B.; Krishnamurthy, V.N.; Bhoraskar, S.V. Humidity sensing with weak acid-doped polyaniline and its composites. Sensors and Actuators B: Chemical, 2003, 96, 124.

7. Joon-Boo, Yu.; Hyung-Gi, B.; Myung-Suk, So.; Jeung-Soo, H. Analysis of diabetic patient's breath with conducting polymer sensor array. Sensors and Actuators B: Chemical, 2005, 108, 305.

8. Gangopadhay, R.; De, A. Conducting polymer composites: novel materials for gas sensing. Sensors and Actuators B: Chemical, 2001, 77, 326.

9. Amrani, M.E.H.; Dowdeswell, R.M.; Payne, P.A.; Persaud, K.C. An intelligent gas sensing system. Sensors and Actuators B: Chemical, 1997, 44, 512.

10. Jouve, C.; Jullien, D.; Remaki, B. Conductive polyethylene as sensitive layer for gas detection. Sensors and Actuators B: Chemical, 1995, 28, 75.

11. Dogan, S.; Akbulut, U.; Yalcin, T.; Suzer, S.; Toppare, L. Conducting polymers of aniline. II. A composite as a gas sensor. Synthetic Metal, 1993, 60, 27.

12. Ram, M.K.; Yavuz, O.; Aldissi, M. $\mathrm{NO}_{2}$ gas sensing based on ordered ultrathin films of conducting polymer and its nanocomposite. Synthetic Metal, 2005, 151, 77.

13. Amado, F.D.R.; Gondran, E.; Ferreira, J.Z.; Rodrigues, M.A.S.; Ferreira, CA. Synthesis and characterization of high impact polystyrene/polyaniline composite membranes for electrodialysis. Journal of Membrane Science, 2004, 234, 139.

14. Gupta, R.K.; Singh, R.A. Preparation and characterization of polymer composites of polyaniline with polyvinylchloride and polystyrene. Journal of Non-Crystalline Solids, 2005, 351, 2022.

15. Srivastava, A.; Singh, T.V.; Mule, S.; Rajan, C.R.; Ponrathnam, S. Study of chemical, optical and thermal modifications induced by $100 \mathrm{MeV}$ silicon ions in a polycarbonate film. Nucl. Instr. Meth. B, 2002, 192, 402. 
16. Hioki, T.; Noda, S.; Sugiura, M.; Kakaeno, M.; Yamada, K.; Kawamoto, J. Electrical and optical properties of ion-irradiated organic polymer Kapton H. Appl. Phys. Lett. 1983, 43, 30.

17. Sevil, U.A.; Güven, O.; Kovács, A.; Slezsák, I. Gamma and electron dose response of the electrical conductivity of polyaniline based polymer composites. Radiation Physics and Chemistry, 2003, 67, 575.

18. Sonar, P.; Sharma, A.L.; Chandra, A.; Muellen, K.; Srivastava, A. Synthesis and study of conductivity behavior of blended conducting polymer films irradiated with swift heavy ions of silicon. Current Applied Physics, 2003, 3, 247.

19. Singh, N.L.; Shrinet, V.; Pandaya, N.R.; Sharma, A.; Patel, N.V.; Avasthi, D.K. Effect of ion irradiation on hydrogen gas sensitivity of polymer. Nucl. Instr. Meth. B., 1999, 156, 191.

20. Ziegler, J.F.; Biersack, J.P. The stopping ranges of ions in matter, SRIM-2003, IBM Research, New York, USA (2000) 1.

21. Meixiang W.; Ming Li.; Junchao L.; Zhenxing L. Structure and electrical properties of the oriented polyaniline films. J. Applied Poly Science. 1994, 53, 131.

(C) 2006 by MDPI (http://www.mdpi.org). Reproduction is permitted for non-commercial purposes. 